\title{
MINIMAL SETS IN RECURRENT DISCRETE FLOWS
}

\author{
RONALD A. KNIGHT
}

\begin{abstract}
Each orbit closure of a recurrent discrete flow on a locally compact space is shown to contain at most one compact minimal subset. In particular, such an orbit closure contains a unique compact minimal subset if and only if the orbit closure is compact.
\end{abstract}

1. Introduction. Katznelson and Weiss constructed a recurrent discrete flow on a compact metric orbit closure in [1] "in which there is a unique minimal set, all points are generic, and there is a continuous arc of ergodic measures." The uniqueness of the compact minimal set is not surprising in view of our theorem and its corollary in $\S 2$.

Throughout this paper we let $(X, T)$ denote a given discrete flow on a locally compact Hausdorff phase space $X$ induced by a homeomorphism $T$ of $X$ onto $X$. A point $x$ of $X$ is recurrent provided $T^{n_{i}}(x) \rightarrow x$ for some sequence $n_{i} \rightarrow+\infty$. (Recurrent points have also been called positively Poisson stable in the literature (see [4]).) When each point of $X$ is recurrent we say $(X, T)$ is recurrent. The orbit, orbit closure, and limit relations on $X$ are denoted, respectively, by $C, K$, and $L$ with each semirelation indicated by the appropriate + or - superscript (see [2 and 3]).

2. Recurrent flows. Our first result implies that the set consisting of the compact minimal sets in any discrete flow is uniformly recurrent whenever the phase space is compact.

PROPOSITION 1. If the phase space $X$ of $(X, T)$ is compact and $V$ is an open set containing each compact minimal set in $X$, then there exists an $N_{v}>0$ such that $X=\bigcup_{n=0}^{N_{V}} T^{n}(V)$.

ProOF. The set $\bigcup\left\{T^{n}(V): n\right.$ an integer $\}$ is an open invariant set, and hence, has a closed invariant complement. Whenever the complement is nonempty it must contain a compact minimal set, and is therefore empty. Thus, $\left\{T^{n}(V):|n|<+\infty\right\}$ is an open cover of $X$ which contains a finite subcover of the form $\left\{T^{n}(V):|n|<N\right\}$ and we can choose $N_{V}=2 N$. The proof is complete.

Next, we state our major results; however, before proving the theorem we shall prove one lemma.

THEOREM. Let $X$ be a compact recurrent orbit closure in $(X, T)$. Then every pair of orbit closures intersect and, in particular, $X$ contains a unique compact minimal set.

Received by the editors May 2, 1985 and, in revised form, August 19, 1985.

1980 Mathematics Subject Classification (1985 Revision). Primary 54H20.

Key words and phrases. Discrete flows, minimal set, recurrent. 
Any two compact minimal orbit closures in $X$ of the theorem intersect, and hence, coincide. The theorem applies to the extended flow on the one point compactification $X \cup\{\infty\}$, whenever the orbit closure $X$ is not compact, and $\{\infty\}$ is the compact minimal subset. Thus, we have the following corollary.

COROLLARY. Each orbit closure in a recurrent flow $(X, T)$ contains at most one compact minimal subset. Furthermore, such an orbit closure contains a unique compact minimal set if and only if the orbit closure is compact.

LEMMA 1. Let $X$ be a compact recurrent orbit closure in $(X, T)$ containing no fixed points. Then any two orbit closures intersect.

ProOF. Suppose $K(x)$ and $K(y)$ are disjoint orbit closures. Select a compact neighborhood $V$ of $K(x)$ such that $K(y) \subset X \backslash V$. We shall proceed by showing that no such set $V$ can exist. Denote $\min \left\{n>0: T^{n}(z) \in V^{0}\right\}$ by $n_{z}$ for each point $z$ of $H=C\left(V^{0}\right) \cap C(X \backslash V)$. Note that, since $X$ is a recurrent orbit closure, $H$ is a nonempty open set and $n_{z}$ is a positive integer. For a compact subset $F$ of $H \cap V$ define $N_{F}$ to be $\operatorname{lub}\left\{n_{z}: z \in F\right\}$. For any point $z$ in $F$ the continuity of $T$ implies $n_{p} \leq n_{z}$ for each point $p$ in some neighborhood $V_{z}$ of $z$. The covering $\left\{V_{z}: z \in F\right\}$ of $F$ contains a finite subcovering $\left\{V_{z_{i}}: i=1, \ldots, k\right\}$. For each point $z$ in $F$ we have $n_{z} \leq \max \left\{n_{z_{i}}: i=1, \ldots, k\right\}$. Hence, $N_{F}$ is finite.

There is a net $\left(x_{i}\right)$ in $V^{\circ} \cap H$ converging to $x$ and a net $n_{i} \rightarrow+\infty$ such that $T^{n_{i}}\left(x_{i}\right) \rightarrow y$. Define $m_{i}$ to be $\max \left\{n: 0<n<n_{i}, T^{n}\left(x_{i}\right) \in V\right\}$. The net $\left(T^{m_{i}}\left(x_{i}\right)\right)$ is in the compact set $V \cap H$, and so, $\left(x_{i}\right)$ and $\left(m_{i}\right)$ can be chosen so that $T^{m_{i}}\left(x_{i}\right)$ converges to a point $p$ of $V \cap \bar{H}$. If $p \in H$, then $H$ contains a compact neighborhood $G$ of $p$. The compact subset $F=G \cap V$ of $V$ ultimately contains the net $\left(T^{m_{i}}\left(x_{i}\right)\right)$ with $T^{n_{i}}\left(x_{i}\right)=T^{n_{i}-m_{i}}\left(T^{m_{i}}\left(x_{i}\right)\right) \in \bigcup\left\{T^{n}(F): 0 \leq n \leq N_{F}\right\}$. Thus, $\left(T^{n_{i}}\left(x_{i}\right)\right)$ converges to $y$ in $\bigcup\left\{T^{n}(F): 0 \leq n \leq N_{F}\right\}$ implying $C(y)$ intersects $V$, which is absurd. Thus, $x \in \bar{H} \backslash H$, and consequently, $p$ must be an element of the boundary of $H$ with either $C(p) \subset V$ or else $C(p) \subset X \backslash V^{0}$ and $C(p) \not \subset \partial V$. Note also that by the way $\left(m_{i}\right)$ was chosen $p \in \partial V \cup T^{-1}(\partial V)$, and hence, $p \notin K(x)$ and $p \notin K(y)$.

Assume $C(p) \subset V$. Either $C(p) \cap V^{0}$ is nonempty or else $C(p) \subset \partial V$. First, consider the case $C(p) \cap V^{0}$ nonempty. Select a compact neighborhood $G$ of $T^{n}(p)$ for some $n<0$ where $T^{n}(p) \neq p, K(x) \subset X \backslash G, p \notin G$, and $G \cap T^{n}(G)=\varnothing$. Let $V_{0}$ denote the compact neighborhood $V \backslash G^{0}$ of $K(x)$. The point $p$ is in $H_{0}=C\left(V_{0}^{0}\right) \cap$ $C\left(X \backslash V_{0}\right)$, and again, relative to $V_{0}$, we have $\left(T^{n_{i}}\left(x_{i}\right)\right)$ ultimately in $\bigcup\left\{T^{n}\left(F_{0}\right): 0 \leq\right.$ $\left.n \leq N_{F_{0}}\right\}$ and $C(y)$ intersects $V$. Consequently, $C(p)$ must be contained in the boundary of $V$. By choosing sets $G$ and $V_{0}$ as we did above, a neighborhood $V_{0}$ is obtained with $C(p) \subset X \backslash V_{0}^{0}$ and $C(p)$ intersects $X \backslash V_{0}$. This situation is the same as our second case in the preceding paragraph, namely, $C(p) \subset X \backslash V^{0}$ but $C(p) \not \subset \partial V$, and hence, it is sufficient to show the second case is impossible in order to obtain our contradiction.

Now, assume $C(p) \subset X \backslash V^{0}$ and $C(p) \not \subset \partial V$. Choose a compact neighborhood $G$ of $p$ such that $K(y) \subset X \backslash G, T^{j}(G) \subset X \backslash V^{0}$ for some $j>0$, and $G \cap T^{j}(G)=$ $\varnothing$. Select a compact neighborhood $W$ of $p$ in $G^{0}$. Define $K_{z}$ for $z$ in $W$ to be $\min \left\{n>0: T^{m}(z) \in X \backslash V \cup G\right.$ for some $0 \leq m \leq n$, and $\left.T^{n}(z) \in G^{0}\right\}$ and define $N$ to be lub $\left\{K_{z}: z \in W\right\}$. By an argument similar to the one above showing $N_{F}$ is finite, $N$ is finite. There is a net $\left(y_{i}\right)$ exterior to $V \cup G$ converging to $y$ such that $C^{-}\left(y_{i}\right) \cap W^{0}$ is nonempty for each $i$ since $p=\lim T^{m_{i}}\left(x_{i}\right)=\lim T^{m_{i}-n_{i}}\left(y_{i}\right)$ with 
$y_{i}=T^{n_{i}}\left(x_{i}\right), y_{i} \rightarrow y$, and $m_{i}-n_{i}<0$. Let $N_{i}=\max \left\{n<0: T^{n}\left(y_{i}\right) \in W^{0}\right\}$. Then, $-N<N_{i}<0$ and $\left(y_{i}\right)$ can be chosen so that $\left(T^{N_{i}}\left(y_{i}\right)\right)$ converges to a point $q$ of $W$. We have $y_{i} \in\left\{T^{n}\left(y_{i}\right): N_{i} \leq n \leq N_{i}+N\right\} \subset\left\{T^{n}\left(y_{i}\right):-N \leq n \leq N\right\} \subset$ $\bigcup_{n=-N}^{N} T^{n}(W)$, and hence, $y \in \bigcup_{n=-N}^{N} T^{n}(W)$. Again, the orbit $C(y)$ intersects $G$ which is absurd. Consequently, no neighborhood with the properties possessed by $V$ exists and the asserted result follows. The proof of Lemma 1 is complete.

PROOF OF THE THEOREM. Let the flow $(\tilde{X}, \tilde{T})$ on $\tilde{X}=X \times\{0,1\}$ be defined by the "square root" $\tilde{T}$ of $T$, i.e., $\tilde{T}(x, i)=\left(T^{i}(x), 1-i\right)$ for $i \in\{0,1\}$ and $x \in X$. If $\rho: \tilde{X} \rightarrow X$ is the projection of $\tilde{X}$ onto $X$, then $\rho \circ \tilde{T}^{2} \mid X \times\{0\}=T$. Lemma 1 applies to $(\tilde{X}, \tilde{T})$, and hence, any two orbit closures in $\tilde{X}$ intersect. Consequently, any two orbit closures in $X$ intersect. The proof of the theorem is complete.

Using "root" constructions as in the proof above, we obtain periodic compact minimal subsets of every positive period and the Katznelson-Weiss example in [1] contains a fixed point. Of course, there are compact minimal orbit closures which are neither fixed nor periodic; however, can such an orbit closure be a proper subset of a recurrent orbit closure? Such flows do exist. For example, let $(X, T)$ denote a recurrent orbit closure with a fixed point $p$ and let $(Y, S)$ denote a regularly almost periodic minimal orbit closure; i.e., for each neighborhood $V$ of any point $y$ of $Y$ there is a positive integer $N$ such that $S^{n N}(y)$ belongs to $V$ for every integer $n$. The orbit closure $K(x, y)$ in the product flow $(X \times Y, T \times S)$ contains the unique compact minimal set $\{p\} \times Y$ whenever $K(x)=X$ and $y \in Y$.

Lemma 3 of $[\mathbf{3}]$ can be generalized to obtain the following lemma with no significant change in the technique of its proof.

LEMMA 2. Let $K(y)$ be a recurrent orbit closure of the discrete flow $(X, T)$. Suppose that a neighborhood $V$ of $K(y)$ exists which contains no complete orbit except those in $K(y)$ and suppose that there do not exist points $p, q \notin K(y)$ such that $L^{+}(p) \subset K(y)$ and $L^{-}(q) \subset K(y)$. Then, either $K(y)$ is asymptotically stable or negatively asymptotically stable, i.e., a stable weak attractor or a negatively stable negative weak attractor (see [3]).

If the compact minimal set $M$ in $X$ of the theorem is not $X$, it is stable in neither the positive nor the negative sense because $(X, T)$ is a recurrent orbit closure. Moreover, there do not exist points $p, q \notin M$ such that $L^{+}(p) \subset M$ and $L^{-}(q) \subset M$ since the flow is recurrent. By Lemma 2, every neighborhood $V$ of $M$ contains a complete orbit outside of $M$. Property (i) of the Katznelson-Weiss example in $[\mathbf{1}$, p. 210] generalizes to the following proposition.

PROPOSITION 2. Let $X$ be a compact recurrent orbit closure in $(X, T)$ and let $M$ be the compact minimal subset of $X$. Either $X=M$ or else there is a net $\left(C\left(y_{i}\right)\right)$ of disjoint orbits in $X \backslash M$ such that $\left(K\left(y_{i}\right)\right)$ is a linear net of orbit closures with intersection $M$.

The author wishes to express appreciation to the referee for suggesting that the "square root" function be used in the theorem in order to strengthen the result, and for carefully reading the paper. 


\section{REFERENCES}

1. Y. Katznelson and B. Weiss, When all points are recurrent/generic, Ergodic Theory and Dynamical Systems. I, Birkhäuser, Boston-Basel-Stuttgart, 1981, pp. 195-210.

2. R. Knight, Prolongationally stable discrete flows, Fund. Math. 108 (1980), 137-144.

3. __ Compact discrete flows, Fund. Math. 118 (1983), 193-190.

4. _ A characterization of recurrent motions, Bull. Austral. Math. Soc. 28 (1983), 1-4.

Division of MAThematics and COMPUTER SCIENCE, NoRTheast Missouri State UNIVERSITY, KIRKSVILLE, MISSOURI 63501 